\title{
EFFICIENT ESTIMATION OF NONSTATIONARY SPATIAL COVARIANCE FUNCTIONS WITH APPLICATION TO HIGH-RESOLUTION CLIMATE MODEL EMULATION
}

\author{
Yuxiao Li and Ying Sun \\ King Abdullah University of Science and Technology
}

\begin{abstract}
Spatial processes exhibit nonstationarity in many climate and environmental applications. Convolution-based approaches are often used to construct nonstationary covariance functions in Gaussian processes. Although convolutionbased models are flexible, their computation is extremely expensive when the data set is large. Most existing methods rely on fitting an anisotropic, but stationary model locally, and then reconstructing the spatially varying parameters. In this study, we propose a new estimation procedure to approximate a class of nonstationary Matérn covariance functions by local-polynomial fitting the covariance parameters. The proposed method allows for efficient estimation of a richer class of nonstationary covariance functions, with the local stationary model as a special case. We also develop an approach for a fast high-resolution simulation with nonstationary features on a small scale and apply it to precipitation data in climate model outputs.

Key words and phrases: Climate model runs, conditional simulation, large datasets, local likelihood estimation, nonstationary Matérn covariance function, polynomial approximation.
\end{abstract}

\section{Introduction}

Gaussian random fields (GRFs) or Gaussian processes (GPs) for spatial data have been called "the most valuable tools in the toolkit for geo-statistical modeling" (Gelfand and Schliep (2016)). These tools are important because the probabilistic distribution of a GRF can be fully determined by its first and second moments. For convenience, GRFs are typically assumed to be stationary or isotropic, which implies that the second moment is finite, the mean function is constant, and a certain property of the covariance function is invariant. Nevertheless, nonstationarity often exists in spatial processes, such as between the land and the ocean, or between mountains and plains. In general, the nonstationarity exists in the mean and the covariance function. In this study, we assume that the mean function is constant to focus on nonstationarity in the covariance function. 
The existing literature provides various approaches to modeling nonstationary covariance functions. We classify these approaches into six groups: 1) basis function expansions (Nychka, Wikle and Royle (2002)); 2) deformation approaches (Sampson and Guttorp (1992); Anderes and Stein (2008)); 3) differential operator approaches (Jun and Stein (2008); Lindgren, Rue and Lindström (2011)); 4) process convolution approaches (Higdon (1998); Paciorek and Schervish (2006)); 5) predictive processes (Gramacy and Lee (2008)); and 6) treed Gaussian processes (Banerjee et al. (2008)). Risser (2016) reviewed most of these methodologies, emphasizing convolution-based methods. The convolution-based model has become one of the most popular methods in spatial nonstationary modeling, owing to its high flexibility. In particular, Paciorek and Schervish (2006) derived a class of valid nonstationary Matérn covariance functions with convolution models. They characterized nonstationarity by spatially varying parameters, which could be viewed as a function over space.

However, if we specify a nonstationary type using spatially varying parameters, the number of parameters to be estimated will be proportional to the size of the locations. Accuracy and efficiency in such an estimation are extremely difficult to achieve. To address this problem, current methods assume that the covariance function is local stationary (Paciorek and Schervish (2006); Anderes and Stein (2011)) or weighted local stationary (Risser and Calder (2015a); Fouedjio, Desassis and Rivoirard (2016)). Although these approximations simplified the model fitting in many applications, they all rely on the assumption that the spatially varying parameters are locally constant or that the process is stationary in each subregion. Therefore, the approximation quality is significantly affected by the choice of region partition, which is not an easy task. In general, an accurate local fitting requires a reasonably large number of observations, which are often collected over a large, and thus possibly nonstationary spatial region. A smaller size subregion tends to be more stationary; however, there might not be enough data to fit the model locally.

To avoid this problem, we propose a local estimation approach for nonstationary covariance functions based on a higher-order polynomial approximation of the spatially varying parameters. Compared with the local stationary model, our higher-order polynomial approximation allows for local nonstationarity and incorporates local stationary and weighted local stationary models as special cases. We also develop an efficient likelihood-based estimation method for model fitting. Additionally, we show that each polynomial parameter measures the degree of a certain type of nonstationarity. 
Fitted nonstationary models have many potential applications. We apply the proposed method to precipitation data in North America from relatively coarseresolution climate model runs. With the fitted model, we perform high-resolution simulations and generate nonstationary precipitation fields. High-resolution nonstationary simulations are also challenging. Kleiber (2016) proposed combining the approaches of circular embedding and deformation to achieve an exact simulation. We propose a different approach using a sequentially conditional simulation. Using a personal computer, it takes approximately 15 minutes to fit our model using five runs of the general circulation model (GCM) data from $13 \times 30=390$ locations and to perform one simulation at the scale of a regional climate model $(\mathrm{RCM})$ at $62 \times 210=13,020$ locations.

The remainder of our paper is organized as follows. Section 2 introduces convolution-based nonstationary spatial covariance modeling and reviews existing estimation methods. Section 3 proposes our new estimation approaches. Section 4 describes the interpretations, computational issues, and implied simulation algorithms. Section 5 presents simulation studies to fit nonstationary Matérn

covariance functions. Section 6 applies our estimation and high-resolution emulation approaches to precipitation data from climate model runs. Section 7 summarizes our main results and suggests directions for future work.

\section{Nonstationary Covariance Function and its Estimation}

\subsection{Nonstationary spatial covariance function}

A univariate Gaussian random field (GRF), $\{Z(\mathbf{s}), \mathbf{s} \in D\}$, defined on $D \subset$ $\mathbb{R}^{d}$, can be specified as

$$
Z(\mathbf{s})=m(\mathbf{s})+Y(\mathbf{s})+\varepsilon(\mathbf{s}), \mathbf{s} \in D,
$$

where $m(\cdot)$ is the mean function, $Y(\cdot)$ is a spatially dependent and zero-mean GRF with covariance function $C(\cdot, \cdot)$, and $\varepsilon(\cdot) \sim N\left(0, \tau^{2}(\cdot)\right)$ is the nugget effect caused by measurement inaccuracy and environmental variability. Moreover, $m(\cdot)$ is assumed to be a constant for simplicity, $C(\cdot, \cdot)=C\left(\cdot, \cdot ; \boldsymbol{\theta}_{0}\right)$ has a parametric form with $\boldsymbol{\theta}_{0}$ in $\mathbb{R}^{d}$, and $\varepsilon(\cdot)$ and $Y(\cdot)$ are independent.

Equation (2.1) is a general representation that allows for nonstationarity. Let $\boldsymbol{\theta}=\left\{\boldsymbol{\theta}_{0}, \boldsymbol{\tau}\right\}$ be a vector of all unknown parameters to be estimated, and let $C(\cdot, \cdot ; \boldsymbol{\theta})$ be the covariance function that incorporates the nugget effect. When assuming an isotropic covariance function, $C^{I}(\cdot ; \boldsymbol{\theta})$, various classes (exponential, Gaussian, Matérn, Cauchy, etc.) of covariance models are available in spatial statistics (Cressie (2015)), with the Matérn function being the most popular. 
However, $C(\cdot, \cdot ; \boldsymbol{\theta})$, as a valid nonstationary covariance function, may not have a closed form, in general, and may involve many spatially varying parameters that require estimation. Based on kernel convolution (Higdon (1998)), Paciorek and Schervish (2004), Stein (2005), and Paciorek and Schervish (2006) provided a rich class of valid parametric nonstationary Matérn covariance functions on $\mathbb{R}^{d}$ :

$$
\begin{aligned}
C^{\mathrm{NS}}\left(\mathbf{s}_{i}, \mathbf{s}_{j} ; \boldsymbol{\theta}\right)= & \tau\left(\mathbf{s}_{i}\right) \mathbb{1}_{[i=j]}\left(\mathbf{s}_{i}, \mathbf{s}_{j}\right)+\sigma\left(\mathbf{s}_{i}\right) \sigma\left(\mathbf{s}_{j}\right)\left|\Sigma\left(\mathbf{s}_{i}\right)\right|^{1 / 4}\left|\Sigma\left(\mathbf{s}_{j}\right)\right|^{1 / 4} \times \\
& \left|\frac{\Sigma\left(\mathbf{s}_{i}\right)+\Sigma\left(\mathbf{s}_{j}\right)}{2}\right|^{-1 / 2}\left(2 \sqrt{\nu Q_{i j}}\right)^{\nu} K_{\nu}\left(2 \sqrt{\nu Q_{i j}}\right),
\end{aligned}
$$

where $\boldsymbol{\theta}$ is a vector of unknown parameters, $\sigma\left(\mathbf{s}_{i}\right)$ is the spatially varying standard deviation (squared root of the partial sill), $\Sigma\left(\mathbf{s}_{i}\right)$ is the $d \times d$ kernel matrix at $\mathbf{s}_{i}$, that controls the spatially varying local anisotropy (including the spatial range and the direction of the anisotropy), $K_{\nu}(\cdot)$ is a modified Bessel function of the second kind, $\nu$ is a smoothness parameter, and $Q_{i j}=\left(\mathbf{s}_{i}-\mathbf{s}_{j}\right)^{T}\left(\left(\Sigma_{i}+\Sigma_{j}\right) / 2\right)^{-1}\left(\mathbf{s}_{i}-\right.$ $\mathbf{s}_{j}$ ) is the Mahalanobis distance between $\mathbf{s}_{i}$ and $\mathbf{s}_{j}$. The smoothness parameter can be spatially varying as well. For example, the model proposed by Stein (2005) allows for nonstationarity in the smoothness by letting $\nu_{i j}=\left(\nu\left(\mathbf{s}_{i}\right)+\nu\left(\mathbf{s}_{j}\right)\right) / 2$. The covariance functions in Equation (2.2) were derived from kernel convolution models (Higdon (1998)). The nonstationarity is controlled by the spatially varying parameters, $\boldsymbol{\theta}\left(\mathbf{s}_{i}\right)=\left\{\Sigma\left(\mathbf{s}_{i}\right), \sigma\left(\mathbf{s}_{i}\right), \tau\left(\mathbf{s}_{i}\right), \nu\left(\mathbf{s}_{i}\right)\right\}$. Usually, kernel matrices are obtained through spectral decomposition. For example, in the case of $d=2$,

$$
\Sigma\left(\mathbf{s}_{i}\right)=\Sigma_{i}=\left[\begin{array}{cc}
\cos \left(\phi_{i}\right) & -\sin \left(\phi_{i}\right) \\
\sin \left(\phi_{i}\right) & \cos \left(\phi_{i}\right)
\end{array}\right]\left[\begin{array}{cc}
\lambda_{1 i} & 0 \\
0 & \lambda_{2 i}
\end{array}\right]\left[\begin{array}{cc}
\cos \left(\phi_{i}\right) & \sin \left(\phi_{i}\right) \\
-\sin \left(\phi_{i}\right) & \cos \left(\phi_{i}\right)
\end{array}\right],
$$

where $\lambda_{1 i}, \lambda_{2 i}>0$ are eigenvalues that represent spatial ranges and $\phi_{i} \in(0, \pi / 2)$ represents the angle of rotation.

Specifically, a GRF is stationary if and only if all of the spatially varying parameters in Equation (2.2) are constant. The isotropic assumption holds if the kernel matrix is a scalar matrix. In principle, all of the parameters could vary spatially, suggesting that there are different types of nonstationarity. However, allowing too many types of nonstationarity is not feasible in practice owing to possible optimization and model identifiability issues (Anderes and Stein (2011)).

\subsection{Likelihood-based covariance estimation}

The maximum likelihood method is appropriate for estimating a GRF model with a specified parametric nonstationary covariance function. Let $Z(\mathbf{s})$ be a GRF with mean $m(\mathbf{s})$ and covariance function $C^{\mathrm{NS}}(\cdot, \cdot ; \boldsymbol{\theta})$, and assume that $m(\mathbf{s})=0$ for simplicity. Suppose that we observe $Z(\mathbf{s})$ at $n$ locations, $\mathbf{s}_{1}, \ldots, \mathbf{s}_{n}$ 
(the locations could be regularly or irregularly spaced). The random vector $\mathbf{Z}=\left\{Z\left(\mathbf{s}_{1}\right), \ldots, Z\left(\mathbf{s}_{n}\right)\right\}^{T}$ follows an $n$-variate Gaussian distribution, that is, $\mathbf{Z} \sim N_{n}\left(\mathbf{0}, \Sigma_{n \times n}^{\mathrm{NS}}\right)$, where the $(i, j)$-th element of the nonstationary covariance matrix, $\Sigma^{\mathrm{NS}}$, is $C^{\mathrm{NS}}\left(\mathbf{s}_{i}, \mathbf{s}_{j} ; \boldsymbol{\theta}(i, j)\right), i, j=1, \ldots, n$. As a result, the corresponding log-likelihood function is

$$
\ell(\boldsymbol{\theta} ; \mathbf{Z})=-\frac{1}{2} \log \left|\Sigma^{\mathrm{NS}}\right|-\frac{1}{2} \mathbf{Z}^{T}\left(\Sigma^{\mathrm{NS}}\right)^{-1} \mathbf{Z}-\frac{n}{2} \log (2 \pi) .
$$

By maximizing the likelihood function in Equation (2.4), we can derive the maximum likelihood estimates (MLEs), $\hat{\boldsymbol{\theta}}$. Note that the mean function is assumed to be zero. If this is not the case, a restricted maximum likelihood method is needed (Patterson and Thompson (1975); Risser and Calder (2015a)). In most environmental studies, only one replicate is available. When independent replicates are obtained, Equation 2.4 can be simply modified by summing the likelihoods for the replicates. However, the main difficulty is that the parameter vector is spatially varying. Paciorek and Schervish (2006) first provided a solution by assuming that each nonstationary parameter varies slowly and regularly over space; that is, $\theta_{i} \approx \theta_{R(i)}$, where $R(i)$ indicates that location $\mathbf{s}_{i}$ falls within subregion $R(i)$, and $\theta_{i}=\theta\left(\mathbf{s}_{i}\right)$, where $\theta\left(\mathbf{s}_{i}\right)$ indicates any nonstationary parameter. By fitting a stationary model in each of $m$ subregions, typically $m \ll n$, they obtain $\hat{\theta}_{R_{(1)}}, \ldots, \hat{\theta}_{R_{(m)}}$. Although the computational burden of this estimation is significantly reduced, this result is not desirable, because both $\theta(\cdot)$ and the fitted surface are discontinuous when they fit spatial models to each subregion.

To overcome this problem, several groups recently proposed methods have been proposed (Risser and Calder (2015a); Fouedjio, Desassis and Rivoirard (2016); Li and Zhu (2016)) to smooth the local stationary estimates. For instance, Risser and Calder (2015a) and Fouedjio, Desassis and Rivoirard (2016) made $\theta(\cdot)$ a discrete mixture of the parameters at representative locations, or anchor locations as Fouedjio, Desassis and Rivoirard (2016) called them. The final estimate of $\theta\left(\mathbf{s}_{i}\right)$ is a smoothly weighted estimate. That is,

$$
\widehat{\theta}\left(\mathbf{s}_{i}\right)=\sum_{k=1}^{m} w\left(\mathbf{s}_{i}, \mathbf{s}_{k}\right) \widehat{\theta}_{k}, \quad w\left(\mathbf{s}_{i}, \mathbf{s}_{k}\right)=\frac{K\left(\mathbf{s}_{i}, \mathbf{s}_{k}\right)}{\sum_{k=1}^{m} K\left(\mathbf{s}_{i}, \mathbf{s}_{k}\right)},
$$

where $m$ is the number of subregions, $\widehat{\theta}_{k}$ is the local stationary estimator in the $k$-th subregion with anchor location $\mathbf{s}_{k}, w\left(\mathbf{s}_{i}, \mathbf{s}_{k}\right)$ is a weight function, and $K(\cdot)$ denotes a $d$-variate kernel function. The Gaussian kernel, $K\left(\mathbf{s}_{i}, \mathbf{s}_{k}\right)=$ $\exp \left(-\left(\left\|\mathbf{s}_{i}-\mathbf{s}_{k}\right\|^{2}\right) / 2 h\right)$, is the most commonly used kernel function, where $h>0$ is the bandwidth parameter. 
Another popular approach to estimate $\theta_{i}$ is moving-window approach: fit a stationary model locally in a small neighborhood of one location, $\mathbf{s}_{i}$, and then move to the next location in a new neighborhood. In this approach, only locations in the neighborhood of $\mathbf{s}_{i}$ are used to estimate $\theta_{i}$, whereas information from locations far away from $\mathbf{s}_{i}$ is ignored. Anderes and Stein (2011) improved the moving-window method by maximizing a weighted local likelihood function that smoothly down-weights faraway locations. Although moving-window methods improve the estimation in some sense, they are extremely difficult to fit when the data set is large.

These approaches are all based on a local stationary or weighted local stationary assumption, implying that parameter estimates are constant in predefined subregions or neighborhoods. For the sake of both model flexibility and inference efficiency, we therefore extend the local stationary approach and propose a model that allows for local nonstationarity. We do so by approximating the spatially varying parameters with a local polynomial.

\section{New Covariance Estimation Approaches}

\subsection{Local polynomial approximation of covariance parameters}

Suppose that $\theta(\mathbf{s})$ is continuously differentiable, with the $\alpha$-th differentiation denoted by $D^{\alpha} \theta$. For a given location, $\mathbf{s}_{\mathbf{0}}=\left(s_{01}, \ldots, s_{0 d}\right) \in D$, a Taylor expansion allows $\theta(\mathbf{s})$ to be expanded at $\mathbf{s}_{\mathbf{0}}$, as follows (Königsberger (2013)):

$$
\theta(\mathbf{s})=\sum_{|\alpha| \leq k} \frac{D^{\alpha} \theta\left(\mathbf{s}_{\mathbf{0}}\right)}{\alpha !}\left(\mathbf{s}-\mathbf{s}_{\mathbf{0}}\right)^{\alpha}+\sum_{|\alpha|=k} h_{\alpha}(\mathbf{s})\left(\mathbf{s}-\mathbf{s}_{\mathbf{0}}\right)^{\alpha},
$$

where $\lim _{\mathbf{s} \rightarrow \mathbf{s}_{\mathbf{o}}} h_{\alpha}(\mathbf{s})=0$. In particular, for any location $\mathbf{s}=\left(s_{1}, s_{2}\right) \in \mathbb{R}^{2}$, the first-order Taylor approximation of $\theta(\cdot)$ at $\mathbf{s}_{0}=\left(s_{01}, s_{02}\right)$ is

$$
\theta\left(s_{1}, s_{2}\right) \approx \beta_{0}+\beta_{1}\left(s_{1}-s_{01}\right)+\beta_{2}\left(s_{2}-s_{02}\right),
$$

where $\beta_{0}=\theta\left(s_{01}, s_{02}\right), \beta_{1}=\partial \theta\left(s_{01}, s_{02}\right) / \partial s_{1}, \beta_{2}=\partial \theta\left(s_{01}, s_{02}\right) / \partial s_{2}$, and the remainder is bounded by $M / 2\left(\left|\mathbf{s}_{1}-\mathbf{s}_{01}\right|+\left|\mathbf{s}_{2}-\mathbf{s}_{02}\right|\right)^{2}$, where $M$ is the upper bound of all second-order partial derivatives of $\theta(\cdot)$.

Equation 3.2 includes local stationarity as a special case. If $\beta_{1}=\beta_{2}=0$, then $\theta\left(s_{1}, s_{2}\right) \approx \theta\left(s_{01}, s_{02}\right)=\beta_{0}$ is a constant in a small neighborhood centered at $\mathbf{s}_{0}$, which implies local stationarity. The estimates of $\beta_{0}$ can be obtained by using the information in the neighborhood of $\mathbf{s}_{0}$. The type of estimator defined in 2.5 is called a locally constant estimator or a Nadaraya-Watson estimator for nonparametric fitting. It can be viewed as an estimator associated with order 
$\alpha=0$ in Equation (3.1). If we assume that $\alpha=1$, the local linear approximation implies that $\theta(\cdot)$ varies linearly. Therefore, by choosing a higher-order, we can model more complex nonstationarity in $\theta(\cdot)$.

Therefore, based on Equation (3.2), we propose a natural nonstationary estimator of $\theta\left(\mathbf{s}_{i}\right)$ at a location, $\mathbf{s}_{i}=\left(s_{i_{1}}, \ldots, s_{i_{d}}\right)$, as an extension of Equation (2.5). For $\theta\left(\mathbf{s}_{i}\right)$ of the form

$$
\theta\left(\mathbf{s}_{i}\right)=\sum_{k=1}^{m} w\left(\mathbf{s}_{i}, \mathbf{s}_{k}\right)\left\{\beta_{0 k}+\sum_{q=1}^{d} \beta_{q k}\left(s_{i_{q}}-s_{k_{q}}\right)\right\}
$$

we estimate it by subsitituting in the MLE of each $\beta_{q k}$, for $q=0, \ldots, d, k=$ $1, \ldots, m$.

The final estimation of $\theta\left(\mathbf{s}_{i}\right)$ is approximated using kernel smoothing in order to smooth the estimations with $m$ different linear trends. The smoothing method is not restricted. Thus many common approaches are viable, such as smoothing spline and Kriging.

In principle, this model can be fitted to any order polynomial approximation with other covariates included. However, for many real-world environmental applications, the first-order model is usually sufficient because, in general, it requires a large amount of local data to make inferences on more complicated nonstationarity. Consequently, we consider only the first-order nonstationarity and develop statistical inference methods.

\subsection{Estimation procedures for nonstationary kernel matrix}

In this subsection, we describe an algorithm for estimating the two-dimensional (2D) spatially varying kernel matrix, $\Sigma\left(\mathbf{s}_{i}\right)$, characterized by three parameters, $\boldsymbol{\theta}_{n s}(\mathbf{s})=\left\{\lambda_{1}(\mathbf{s}), \lambda_{2}(\mathbf{s}), \phi(\mathbf{s})\right\}$, as in Equation 2.3$)$. Other parameters are set to be stationary (constant) and are denoted by $\boldsymbol{\theta}_{\boldsymbol{s}}$.

To estimate $\boldsymbol{\theta}_{n s}(\mathbf{s})$, we need to maximize the full likelihood, as in Equation (2.4), with $\Sigma^{\mathrm{NS}}$ specified as in Equations (2.2) and (3.3). To improve the computational efficiency, we propose an independent likelihood estimation method in which we assume the subregions are independent of each other. Here, we estimate $\boldsymbol{\beta}_{k}, k=1, \ldots, m$, for each subregion independently. The independent log-likelihood is of the form

$$
\tilde{\ell}\left(\boldsymbol{\beta}_{1}, \ldots, \boldsymbol{\beta}_{m} ; \mathbf{Z}\right)=-\frac{1}{2} \sum_{k=1}^{m} \log \left|\Sigma_{k}^{\mathrm{NS}}\right|-\frac{1}{2} \sum_{k=1}^{m} \mathbf{Z}_{k}^{T}\left(\Sigma_{k}^{\mathrm{NS}}\right)^{-1} \mathbf{Z}_{k}-\frac{n}{2} \log (2 \pi),
$$

where $\Sigma_{k}^{\mathrm{NS}}$ parameterized by $\boldsymbol{\beta}_{k}$ is the covariance matrix and $\mathbf{Z}_{k}$ is the data 
vector corresponding to the locations in the $k$-th subregion.

The independent likelihood method significantly improves the computational efficiency, because the local fitting involves fewer parameters and the computation can be easily parallelized. However, the method may be less accurate at boundary locations.

For comparisons with other methods, we call the local stationary and weighted local stationary estimators S0 and WS0 estimators, respectively, because they correspond to constant (zero-order) approximations, and we call our estimator the first-order nonstationary (NS1) estimator. The estimation procedure is as follows:

1) Divide the region into $m$ overlapping or non-overlapping subregions. Denote the partial random vector that includes all locations in the $k$-th subregion as $\mathbf{Z}_{k}, k=1, \ldots, m$.

2) Select the anchor locations, $\mathbf{s}_{1}, \ldots, \mathbf{s}_{m}$, corresponding to the $m$ subregions. Then, fit a local stationary model to obtain the stationary and local stationary (S0) estimators $\boldsymbol{\theta}_{s}$ and $\boldsymbol{\beta}_{0 k}=\left(\beta_{0 k}^{\lambda_{1}}, \beta_{0 k}^{\lambda_{2}}, \beta_{0 k}^{\phi}\right), k=1, \ldots, m$, by maximizing the independent likelihood $\tilde{\ell}\left(\boldsymbol{\beta}_{01}, \ldots, \boldsymbol{\beta}_{0 m}, \boldsymbol{\theta}_{s} ; \mathbf{Z}\right)$.

3) Substitute the local stationary (S0) estimators into Equation (3.3) for each spatially varying parameter. For example, denote $\lambda_{1 k}\left(\mathbf{s}_{i}\right)=\hat{\beta}_{0 k}^{\lambda_{1}}+\sum_{q=1}^{2} \beta_{q k}^{\lambda_{1}}\left(s_{i_{q}}-\right.$ $\left.s_{k_{q}}\right)$. Then, estimate the slope parameters, $\boldsymbol{\beta}_{1 k}=\left(\beta_{1 k}^{\lambda_{1}}, \beta_{1 k}^{\lambda_{2}}, \beta_{1 k}^{\phi}\right)$ and $\boldsymbol{\beta}_{2 k}=$ $\left(\beta_{2 k}^{\lambda_{1}}, \beta_{2 k}^{\lambda_{2}}, \beta_{2 k}^{\phi}\right), k=1, \ldots, m$, by maximizing the independent likelihood $\tilde{\ell}\left(\boldsymbol{\beta}_{11}\right.$, $\left.\ldots, \boldsymbol{\beta}_{1 m}, \boldsymbol{\beta}_{21}, \ldots, \boldsymbol{\beta}_{2 m} ; \mathbf{Z}, \hat{\boldsymbol{\beta}}_{01}, \ldots, \hat{\boldsymbol{\beta}}_{0 m}, \hat{\boldsymbol{\theta}}_{s}\right)$.

4) Smooth the estimators in steps 2 and 3 using Equation (3.3). For example, $\hat{\lambda}_{1}\left(\mathbf{s}_{i}\right)=\sum_{k=1}^{m} w\left(\mathbf{s}_{i}, \mathbf{s}_{k}\right)\left\{\hat{\beta}_{0 k}^{\lambda_{1}}+\sum_{q=1}^{2} \hat{\beta}_{q k}^{\lambda_{1}}\left(s_{i_{q}}-s_{k_{q}}\right)\right\}$. Finally, the first-order nonstationary (NS1) estimator of the kernel matrix is the approximate estimations of all nonstationary parameters $\hat{\boldsymbol{\theta}}_{n s}\left(\mathbf{s}_{i}\right)$ at each location $\mathbf{s}_{i}$.

\section{Interpretations and Computations}

\subsection{Computational issues}

With 2D data, six parameters can exhibit nonstationarity at a given anchor location: three in the kernel matrix, one in the partial sill, one in the smoothness, and one in the nugget effect. For both the full likelihood and the independent likelihood methods, if we allow all of the six parameters to have first-order nonstationarity, then the number of parameters to be estimated is $3 \times 6 \times m$. Usually, $m \ll n$. The number of parameters to be estimated therefore increases with the number of anchor locations $m$ and is less than $n$. 
Obviously, the full likelihood method is more accurate than the independent likelihood method, especially near the boundary between subregions. However, this could lead to optimization issues because too many parameters need to be optimized simultaneously. Using the independent likelihood approach, the parameters can be optimized in parallel and locally. Instead of solving a highdimensional problem, we solve several lower-dimensional problems. Although our method may not reach the global maxima, the optimization is more stable.

Step 2 estimates both the stationary and the nonstationary parameters. The maximization is not trivial when the stationary parameters are unknown, but it can be well fitted by the NSconvofit function in the $\mathrm{R}$ package convoSPAT. Details of their algorithms can be found in Risser and Calder (2015a).

The weight function depends on the bandwidth, $h$. Because our method relies on the NSconvofit function, we choose the same default bandwidth as in the function, which is the square of half the distance between the two closest anchor locations, (i.e., $h=\left\{\left\|\mathbf{s}_{1}-\mathbf{s}_{2}\right\| / 2\right\}^{2}$ ). As a result, the local estimates depend only on the data in the subregions with range $2 \sqrt{h}$, indicating the range of high-density points in a Gaussian distribution. However, other choices can be made using cross-validation, which we discuss in the Supplementary Material (Section S1.1).

The optimization is performed using the optim function in R. With the approximated Hessian matrix, we quantify the uncertainty of our estimations using the asymptotic standard errors and the corresponding confidence intervals.

\subsection{Covariates and degree of nonstationarity}

When the same kernel function, $K(\cdot)$, and bandwidth parameter, $h$, as defined in Equation (2.5), are selected, it is easy to see that $\widehat{\theta}\left(\mathbf{s}_{i}\right)$ in Equation (3.3) is a weighted local stationary estimator when $\beta_{1 k}, \ldots, \beta_{d k}=0$. Moreover, in the $k$-th subregion, $\boldsymbol{\beta}$ can be viewed as the regression parameters of the covariate 1 for $q=0$, and of the covariates $s_{i_{q}}-s_{k_{q}}$ for $q=1, \ldots, d$. Hence, our model can be extended to include additional covariates in a similar way to the regression-based nonstationary model proposed by Risser and Calder (2015b), with all parameters identifiable.

Compared with the local stationary model, the additional parameters in Equation (3.3), $\left\{\beta_{q k}\right\}_{q=1}^{d}$ for $k=1, \ldots, m$, have useful interpretations as measurements of nonstationarity. To illustrate this, we first define the degree of nonstationarity.

Definition 1. Let $\theta(\mathbf{s}), \mathbf{s}=\left(s_{1}, \ldots, s_{d}\right) \in \mathbb{R}^{d}$, be a spatially varying parameter. 
Define the $r$-th order nonstationarity index of $\theta(\mathbf{s})$, denoted by $D_{r}$, as

$$
D_{r}=\frac{1}{d} \sum_{q=1}^{d}\left|\int_{s_{q}} \frac{\partial^{r} \theta(\mathbf{s})}{\partial s_{q}^{r}} d s_{q}\right| .
$$

In particular, we call $D_{1}$ the trend-nonstationary index and $D_{2}$ the wigglinessnonstationary index.

In Definition 1, large values of $D_{r}$ are associated with stronger nonstationarity. For example, under stationarity, $\theta(\mathbf{s})$ is a constant at any location s. As a result, we obtain $D_{r}=0$, which indicates the smallest nonstationarity. In contrast, if $D_{r}$ is large and far from zero, then $\theta(\mathbf{s})$ deviates from the stationary model along at least one direction of $\mathbf{s}$. This definition gives an appropriate and convenient measure for the degree of the $r$-th order nonstationarity.

Specifically, when $r=1$ and $d=1, D_{1}$ is an overall measure of the slope. When $D_{1}$ increases, the spatially varying parameter, $\theta(s)$, changes at a faster rate, and we expect a larger difference between $\theta\left(s_{1}\right)$ and $\theta\left(s_{2}\right)$ at two given locations $s_{1}$ and $s_{2}$. To avoid confusion with the trend of $\theta(s)$ in $s$, we call $D_{1}$ the trend-nonstationary index. Similarly, the wiggliness-nonstationary index, $D_{2}$, is associated with how much these differences change. A related idea for time-series modeling can be found in Das and Nason (2016).

Now, it is easy to see that the parameters of interest, $\left\{\beta_{q k}\right\}_{q=1}^{d}, k=1, \ldots, m$, directly define the trend-nonstationary index, $D_{1}$. In our model, when $s \in[0,1]$ and $m=1$, we have $\theta(s) \approx \beta_{01}+\beta_{11}\left(s-s_{1}\right)$ and $D_{1}=\left|\int_{0}^{1} \mathrm{~d} \theta(s) / \mathrm{d} s d s\right| \approx\left|\beta_{11}\right|$.

In general, in the $k$-th subregion and assuming that $\mathbf{s} \in[0,1]^{d}$, we propose using an empirical estimator of $D_{1}$, defined as $\hat{D}_{1 k}$, to measure the nonstationarity. That is,

$$
\hat{D}_{1 k}=\frac{1}{d} \sum_{q=1}^{d}\left|\hat{\beta}_{q k}\right|, k=1, \ldots, m .
$$

\subsection{Implication for high-resolution emulation}

Estimation and emulation (simulation) are closely linked. Using the estimated parameters, we can directly generate realizations at $N$ new locations, where $N \gg n$, after fitting our model at $n$ observed locations, following the method in Section 3.2. In the 2D situation, let $\left\{\hat{\beta}_{0 k}^{\theta}\right\}_{k=1}^{m},\left\{\hat{\beta}_{1 k}^{\theta}\right\}_{k=1}^{m}$, and $\left\{\hat{\beta}_{2 k}^{\theta}\right\}_{k=1}^{m}$ be the MLEs for a spatially varying parameter, $\theta(\mathbf{s})$. Then, $\theta(\mathbf{s})$ at an unknown location, $\mathbf{s}^{\text {new }}=\left\{s_{1}^{\text {new }}, s_{2}^{\text {new }}\right\}$, is simply specified as 


$$
\hat{\theta}\left(\mathbf{s}^{\text {new }}\right)=\sum_{k=1}^{m} w\left(\mathbf{s}^{\text {new }}, \mathbf{s}_{k}\right)\left\{\hat{\beta}_{0 k}^{\theta}+\sum_{q=1}^{2} \hat{\beta}_{q k}^{\theta}\left(s_{q}^{\text {new }}-s_{k_{q}}\right)\right\},
$$

where $\mathbf{s}_{k}=\left(s_{k_{1}}, s_{k_{2}}\right)$ is the anchor location at the $k$-th subregion. Hence, the new covariance matrix for $\mathbf{s}_{i}^{\text {new }}, i=1, \ldots, N$, formed by the nonstationary Matérn in Equation 2.2), will be of size $N \times N$.

Statistical simulations have a much lower computational burden than estimations do. However, a simulation is challenging if we need to simulate highresolution realizations where $N$ is very large. Early studies of high-resolution simulation (Gneiting et al. (2006); Wood and Chan (1994)) typically focused on stationary or isotropic situations. Only a few studies considered the fast simulation of nonstationary GRFs. Nychka et al. (2015) introduced an idea based on Gaussian Markov random fields (GMRFs) and spatial autoregressive (SAR) models for nonstationary processes. Kleiber (2016) proposed an efficient nonstationary simulation method based on spatial deformation. However, it is computationally expensive to estimate the deformation function before performing the simulation.

We propose an efficient algorithm based on a sequentially conditional simulation. The sequential Gaussian simulation has been widely used in geostatistical simulations (Gotway and Rutherford (1994); Fredericks and Newman (1998)). The idea is to simulate realizations only on a subset at a time. In our application, we simulate one data set for a subregion. Then, the next subregion is simulated, conditioning only on all or part of previously simulated subregions. Further details can be found in the Supplementary Materials (Section S1.2).

\section{Simulation Study}

To investigate the performance of our estimation approach, we consider a simple example where nonstationarity exists only in the variance (partial sill), $\sigma^{2}(\mathbf{s})$. Let $W(\mathbf{s})$ be a stationary GRF with a known covariance function, and let $\sigma(\mathbf{s}): \mathbb{R}^{d} \rightarrow \mathbb{R}^{+}$be an unknown function. Then, $Z(\mathbf{s})=\sigma(\mathbf{s}) W(\mathbf{s})$ defines a GRF with a nonstationary variance. For simplicity, we consider the one-dimensional (1D) case with gridded data, $\sigma(s): \mathbb{R} \rightarrow \mathbb{R}^{+}$. A similar example is examined by Anderes and Stein (2011).

In our example, we set $(\tau, \nu, \lambda)=(0,1,0.2)$ in Equation 2.2) and let $\sigma(s)=$ $2 \sin (s / 0.15)+2.8, s \in[0,1]$. Figure S8 in the Supplementary Material shows one realization of $Z(s)$ and its increments. The pattern matches the true curve 

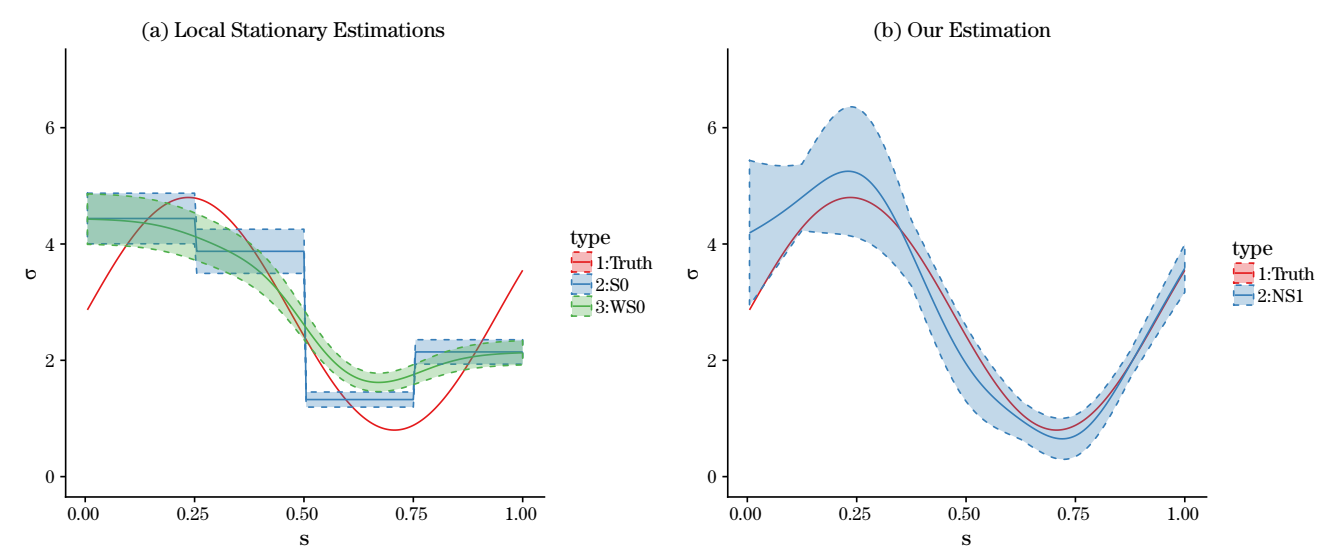

Figure 1. Estimations of $\sigma(s)=2 \sin (s / 0.015)+2.8$ with $95 \%$ confidence intervals using (a) local stationary model (S0) and weighted local stationary (WS0) model and (b) our first-order nonstationary (NS1) model.

of $\sigma(s)$ in Figure 1. Using the algorithm discussed in Section 3.2, we divide the region evenly into four subregions and choose the anchor location as the central point of each subregion. We generate 200 observations at equally spaced locations, with 50 observations in each subregion. Here, only one parameter, $\sigma(s)$, is nonstationary.

As expected, in Figure 1, the estimation using our proposed model is the closest of the models to the truth and the performance is much better than that of the S0 and WS0 models. The corresponding mean squared errors (MSEs) for S0, WS0, and NS1 are 0.54, 0.47, and 0.12, respectively. The more accurate estimation using our method comes at the cost of the largest uncertainty, which is especially significant at the boundaries. In contrast, at the center of each subregion, the uncertainty is minimized.

Approximated confidence intervals give us a way to quantify the uncertainty with one realization. To better measure the uncertainties, we repeated the simulation 100 times. In Table 1, we show the mean, standard error, and trendnonstationary index, $D_{1}$, from 100 local stationary and slope estimators, $\hat{\beta}_{0}$ and $\hat{\beta}_{1}$, respectively. We also calculate the MSEs of $\hat{\sigma}(s)$ from three models based on the mean of $100 \hat{\beta}_{0}$ and $\hat{\beta}_{1}$.

To visualize 100 estimated curves of $\sigma(s)$, we employ functional boxplots (Sun and Genton (2011)), as shown in Figure 2. Here, only WS0 and NS1 models are compared because the S0 model performs worst. In the functional boxplot, the black line indicates the most representative estimate of $\sigma(s)$ from among the 100 
Table 1. Summary of results from 1,000 estimations. Definitions of columns: (1)-(2) The mean (standard deviation) of the S0 estimators $\hat{\beta}_{0}$ and slope estimators $\hat{\beta}_{1} ;$ (3) the empirical trend-nonstationary index in the $k$-th subregion based on the mean of $\hat{\beta}_{0} \mathrm{~S}, \hat{D}_{1 k} ;(4)$ the MSE of $\hat{\sigma}(s), \mathrm{MSE}_{\mathrm{NS} 1}$, calculated from the mean of $\hat{\beta}_{0}$ and $\hat{\beta}_{1}$ from the NS1 model with the corresponding ratios of $\mathrm{MSE}_{\mathrm{S} 0}$ and $\mathrm{MSE}_{\mathrm{WS} 0}$ to $\mathrm{MSE}_{\mathrm{NS} 1} ;$ (5) $\mathrm{MSE}_{\mathrm{S} 0} / \mathrm{MSE}_{\mathrm{NS} 1} ;(6) \mathrm{MSE}_{\mathrm{WS} 0} / \mathrm{MSE}_{\mathrm{NS} 1}$.

\begin{tabular}{|c|c|c|c|c|}
\hline Subregion & 1 & 2 & 3 & 4 \\
\hline \multicolumn{5}{|c|}{$\sigma(s)=2 \sin (s / 0.015)+2.8$ (nonstationary case) } \\
\hline$\hat{\beta}_{0}$ & $4.19(0.43)$ & $3.92(0.43)$ & $1.38(0.19)$ & $2.24(0.28)$ \\
\hline$\hat{\beta}_{1}$ & $9.08(6.18)$ & $-11.37(5.49)$ & $-5.21(1.97)$ & $11.41(2.98)$ \\
\hline$\hat{D}_{1 k}$ & 9.08 & 11.37 & 5.21 & 11.41 \\
\hline $\mathrm{MSE}_{\mathrm{NS} 1}$ & \multicolumn{4}{|c|}{0.050} \\
\hline $\mathrm{MSE}_{\mathrm{S} 0} / \mathrm{MSE}_{\mathrm{NS} 1}$ & \multicolumn{4}{|c|}{9.397} \\
\hline $\mathrm{MSE}_{\mathrm{WS} 0} / \mathrm{MSE}_{\mathrm{NS} 1}$ & \multicolumn{4}{|c|}{8.065} \\
\hline \multicolumn{5}{|c|}{$\sigma(s)=2$ (stationary case) } \\
\hline$\hat{\beta}_{0}$ & $2.01(0.20)$ & $2.01(0.20)$ & $1.99(0.20)$ & $2.00(0.20)$ \\
\hline$\hat{\beta}_{1}$ & $0.00(1.96)$ & $-0.02(1.99)$ & $-0.02(1.42)$ & $-0.02(1.69)$ \\
\hline$\hat{D}_{1}$ & 0.00 & 0.02 & 0.02 & 0.02 \\
\hline $\mathrm{MSE}_{\mathrm{NS} 1}$ & \multicolumn{4}{|c|}{$6.463 e^{-5}$} \\
\hline $\mathrm{MSE}_{\mathrm{S} 0} / \mathrm{MSE}_{\mathrm{NS} 1}$ & \multicolumn{4}{|c|}{0.942} \\
\hline $\mathrm{MSE}_{\mathrm{WS} 0} / \mathrm{MSE}_{\mathrm{NS} 1}$ & \multicolumn{4}{|c|}{1.199} \\
\hline
\end{tabular}
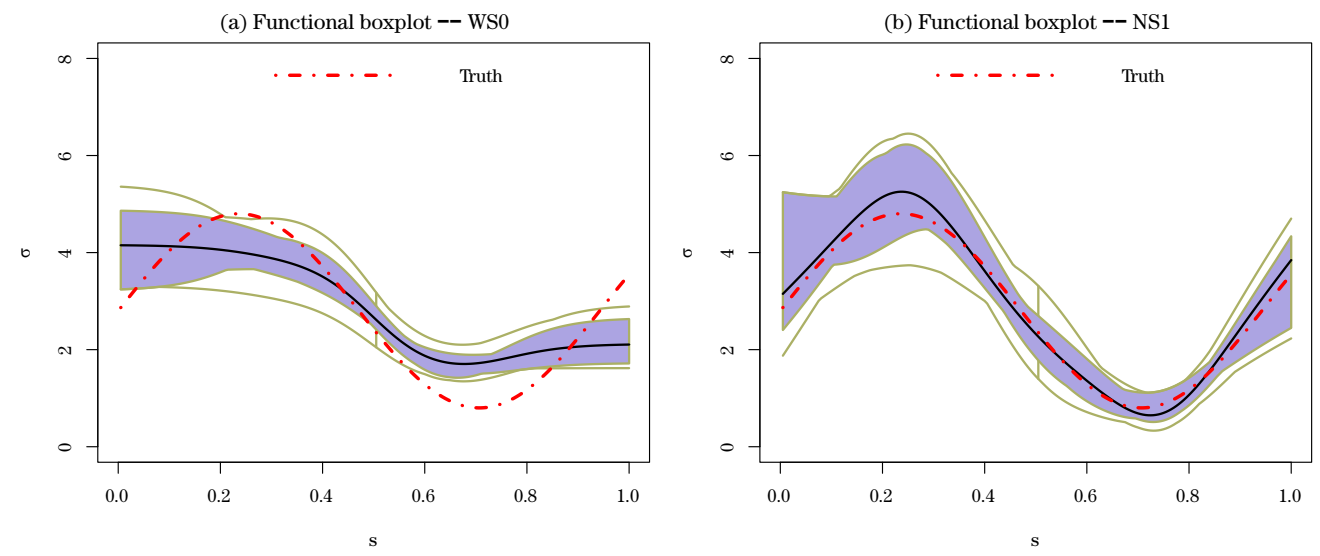

Figure 2. Functional boxplots of the 100 estimated curves of $\sigma(s)$ based on (a) WS0 model and (b) NS1 model.

simulations, and the box (shaded area) shows the variability. We can see that the NS1 model provides the most accurate estimates and outperforms the WS0 model.

The result can be understood in the following way. In general, $\sigma(s)$ increases 
in the first subregion and decreases in the second one, but this change cannot be captured by local constant estimators. If we divide the region into finer subregions, then all of the methods perform better. When estimating $\sigma(s)$, both WS0 and NS1 estimate $\sigma(s)$ as a weighted average of $\hat{\sigma}_{k}$. For the central location in each subregion, the estimate is dominated by the corresponding $\hat{\sigma}_{k}$, but this is not the case for the boundary locations. For example, when $s$ falls in subregion $k$, but close to subregion $k^{\prime}$, the estimation is dominated by both $\hat{\sigma}_{k}$ and $\hat{\sigma}_{k^{\prime}}$, although the observation at $s$ contributes to $\hat{\sigma}_{k}$ only.

When the true underlying GRF is stationary, we expect $\hat{\beta}_{1}$ to be close to zero. Therefore, using the same setup, we simulate data from a stationary GRF with $\sigma(s)=2$. The estimation results in Table 1 clearly show that, under stationarity, the estimated trend-nonstationary index, $\hat{D}_{1 k}$, and the MSEs from all models in each subregion are close to zero. The S0 model in this case gives the best result, but our model provides a similar MSE. Hence, even if our NS1 model is designed to estimate complex nonstationary GRFs, it can also provide satisfactory results when the true model is stationary.

Additional simulation studies are conducted to investigate the sensitivity of the estimation to region division and the computational time, as well as the $2 \mathrm{D}$ estimation with further nonstationary parameters. Detailed results can be found in the Supplementary Material (Section S2). The simulation results show that our model is less sensitive to region division than are the WS0 and S0 models. However, an appropriate region division is still crucial to achieving a good estimation. In general, too few subregions lead to a poor estimation, while too many subregions increase the estimation uncertainty. For example, in the $1 \mathrm{D}$ simulation, two subregions $(m=2)$ are not sufficient, but $m=4,8,10$ are helpful. However, the uncertainty increases too much when $m=8,10$, because there are not enough data for the local fitting. In terms of the computations, more subregions and fewer nonstationary parameters reduce the running time, in general. For the same number of subregions, because our model has a greater number of parameters to estimate, it requires more computational time than the other two rough approximation methods do. However, it is faster than the conventional moving-window method when the window size is the same as the size of the subregion. In the $2 \mathrm{D}$ simulation study, our model still performs the best, although the result is not as significant as in the 1D case.

\section{Application to Climate Model Emulation}

Our application focuses on estimating and simulating data products obtained 
from general circulation model (GCM) outputs. GCMs are useful for forecasting weather and climate changes, but their resolution is too coarse to characterize local patterns. A well-known technique used to produce high-resolution realizations is downscaling (Wilby and Wigley (1997)), such as regional climate model (RCM). Driven by GCM, RCM simulations can include more physical dynamics using local information, such as local humidity, wind speed, and other atmospheric variables. However, local information is not always available. In addition, the process to produce the downscaling data is based on physical approaches. These often involve solving complicated partial differential equations, and thus are time consuming and cannot be completed on personal computers.

In contrast to the RCM, we use our efficient simulation methods to generate high-resolution outputs at the RCM scale. In doing so, we hope that some of the fine-scale statistical properties we observed from the RCM output, particularly the local nonstationarity, can be reproduced. We call the downscaling outputs runs, and our simulated outputs emulations. Without knowing the additional local information, we cannot easily reproduce the complicated local and regional features from RCM runs. However, we are able to reproduce some types of covariance nonstationarity, such as the spatial range and variance.

In this study, we fit third-generation coupled global climate model runs from the Canadian Centre for Climate Modeling and Analysis (CCCma CGCM3 T47, Scinocca and McFarlane (2004)) and consider the corresponding RCM it drives: Canadian RCM (CRCM Version 4.2.3, Shrestha et al. (2014)). The spatial resolution for the GCM runs is about $3.75^{\circ}$ for both latitude and longitude, whereas the RCM runs have much finer resolutions with a $45 \mathrm{~km}$ horizontal mesh (less than $1^{\circ}$ ).

The data of interest are seasonal average precipitation rates for the period 1971 to 2000 in a rectangular region in which the longitude ranges from $157.71^{\circ} \mathrm{W}$ to $35.72^{\circ} \mathrm{W}$ and the latitude ranges from $19.45^{\circ} \mathrm{N}$ to $70.26^{\circ} \mathrm{N}$ (see Figure S9 in the Supplementary Material). Spatially, there are 390 locations (longitude $\times$ latitude $=30 \times 13$ ) for each GCM run. We first transform the data using square-root transformations to reduce the non-Gaussianity. Then, we detrend the transformed data by removing the 30 -year average. Here, to examine the different nonstationarities over time, we choose four reference periods, including two years, a non-El Niño year (1971) and an El Niño year (1998), with two seasons, summer (JJA) and winter (DJF), for each year. Figure S10 in the Supplementary Material shows the preprocessed precipitation rate residuals in summer 1971 from five GCM runs as our observations, and one RCM run as our 

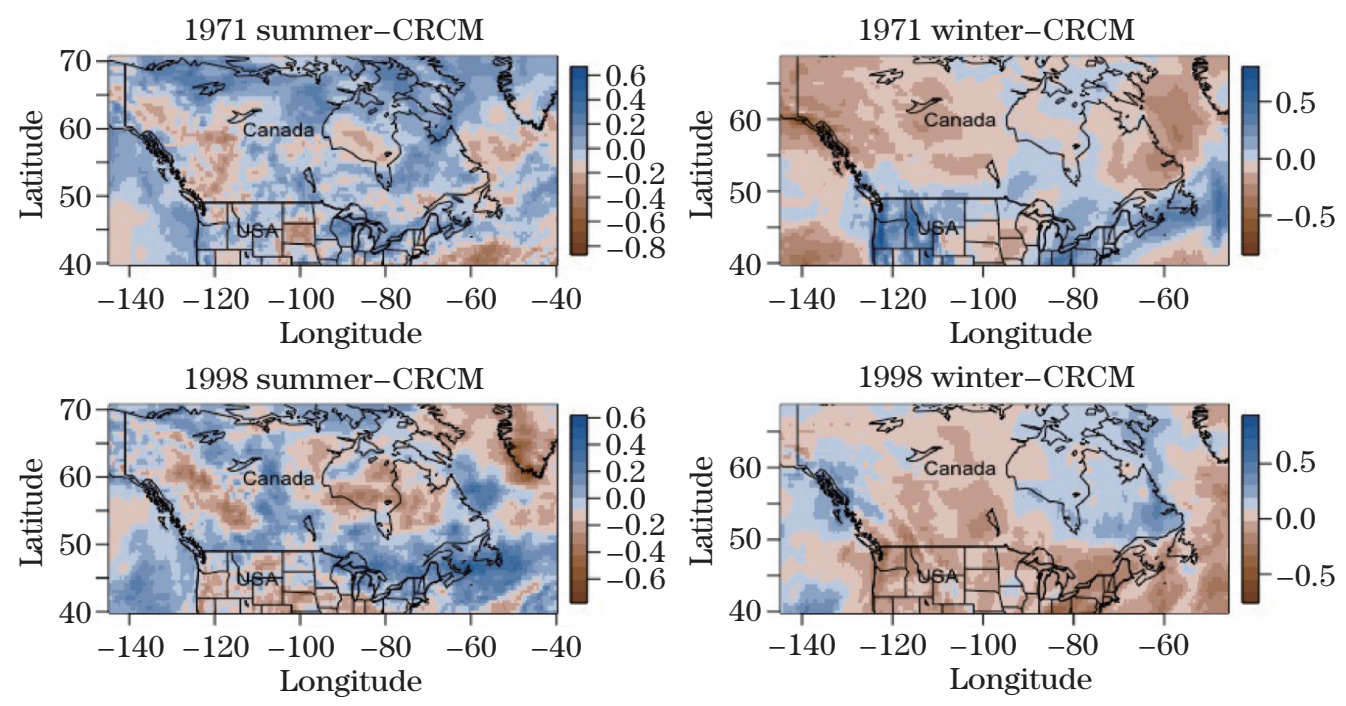

Figure 3. Preprocessed precipitation rate residuals ( $\mathrm{mm} /$ day): four RCM runs in four reference periods.

benchmark. Figure 3 shows the preprocessed precipitation rate residuals from the RCM runs in four percoids.

In this estimation procedure, we scale the coordinates down to $[0,1]^{2}$ and divide the region equally into four subregions, as shown in Figure S4, with four anchor locations chosen at the center of each subregion. We model $\left(\lambda_{1}, \lambda_{2}, \phi\right)$ in the kernel matrix as spatially varying parameters and estimate these parameters, along with others in the Matérn covariance function, treating the five GCM runs as independent replicates. The estimation results of the two spatial ranges $\left(\lambda_{1}, \lambda_{2}\right)$ from the WS0 and NS1 models for the four periods of interest are shown in Figure 4. To see the similarities with the RCM runs, we interpolate the spatially varying parameters to the RCM scale using Equation 4.3.

Visually, a larger spatial range is associated with a more homogenous spatial pattern of the observations. We observe that in the RCM run (Summer 1971), as shown in Figure 3, the north and southeast regions are more homogenous. This agrees with our estimation in Figure 4, where both regions show larger estimated spatial range parameters.

By examing at the nonstationarity over time from the NS1 results in Figure 4 , we see clear nonhomogeneity among seasons. In general, the spatial range is greater during winter, indicating fewer rapid fluctuations over space, which is comparable with the RCM runs. However, the nonstationarity of spatial ranges 

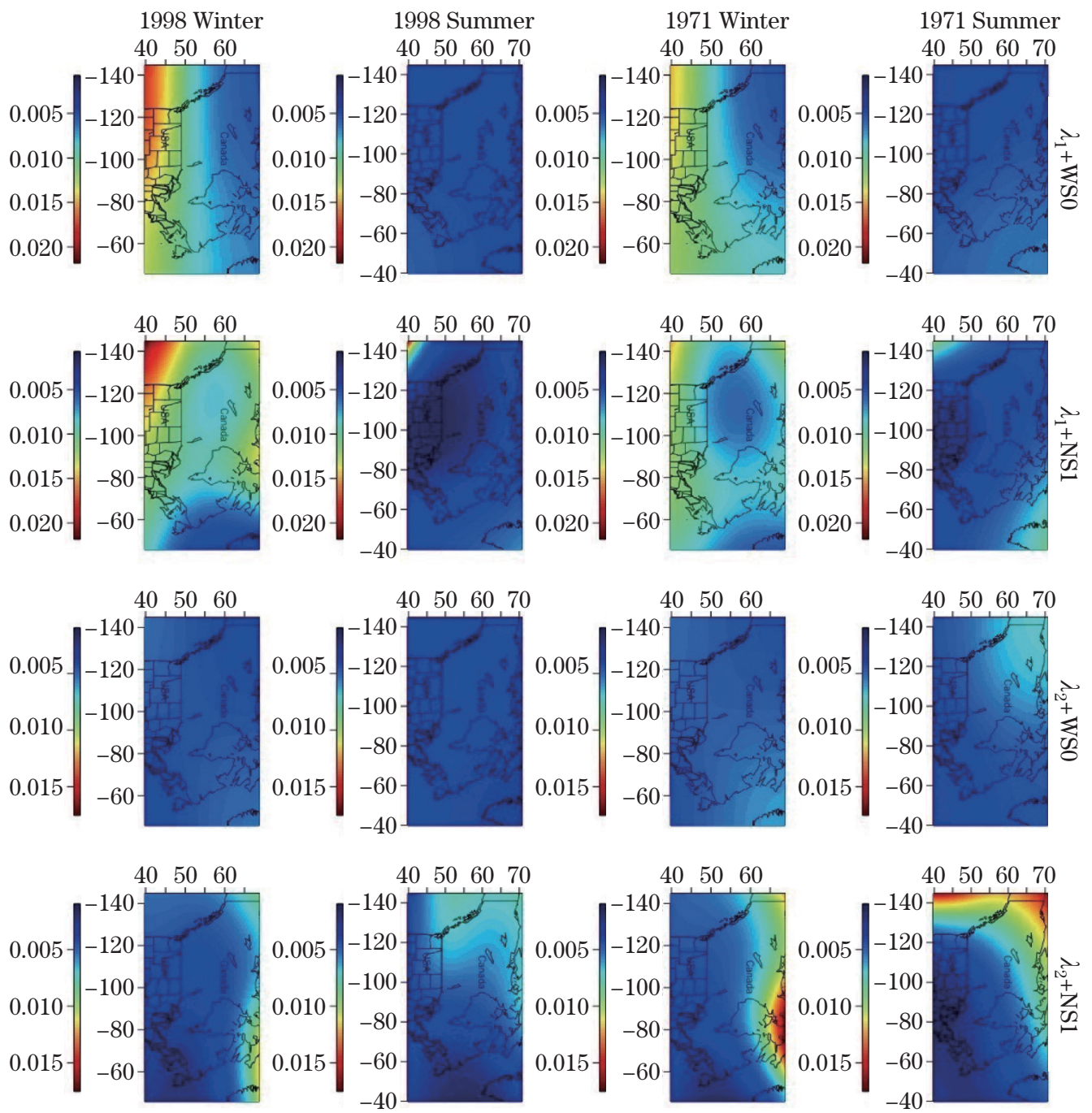

Figure 4. Plots of the estimated spatial ranges, $\lambda_{1}, \lambda_{2}$, based on two models, WS0 and NS1, at four reference periods, 1971 summer, 1971 winter, 1998 summer, and 1998 winter.

is less sensitive among different years for a given season. We can only see the larger spatial ranges out to sea along the Pacific Ocean in the United States and Greenland in Figure 4, indicating that there is nonstationarity in the land-ocean areas. Although the WS0 model also captures some of these characteristics, the estimation is too rough to observe changes in the spatial range at a finer scale.

We also estimate the trend-nonstationary index, $D_{1}$, defined in $(4.2)$. Table 2 
Table 2. Estimated trend-nonstationary index, $\hat{D}_{1 k}, k=1, \ldots, 4$.

\begin{tabular}{|ccc|}
\hline Season & 1971 Summer & 1998 Summer \\
\hline$\hat{D}_{1 k}^{\lambda_{1}}$ & $(4.598,0.855,0.258,1.156)$ & $(11.232,1.570,0.148,1.185)$ \\
$\hat{D}_{1 k}^{\lambda_{2}}$ & $(4.122,0.332,1.622,2.525)$ & $(3.616,0.056,1.322,3.382)$ \\
\hline Season & 1971 Winter & 1998 Winter \\
\hline$\hat{D}_{1 k}^{\lambda_{1}}$ & $(0.696,0.647,2.658,1.891)$ & $(1.037,0.155,2.493,4.156)$ \\
$\hat{D}_{1 k}^{\lambda_{2}}$ & $(0.813,0.048,2.601,6.221)$ & $(0.777,0.262,2.279,4.164)$ \\
\hline
\end{tabular}
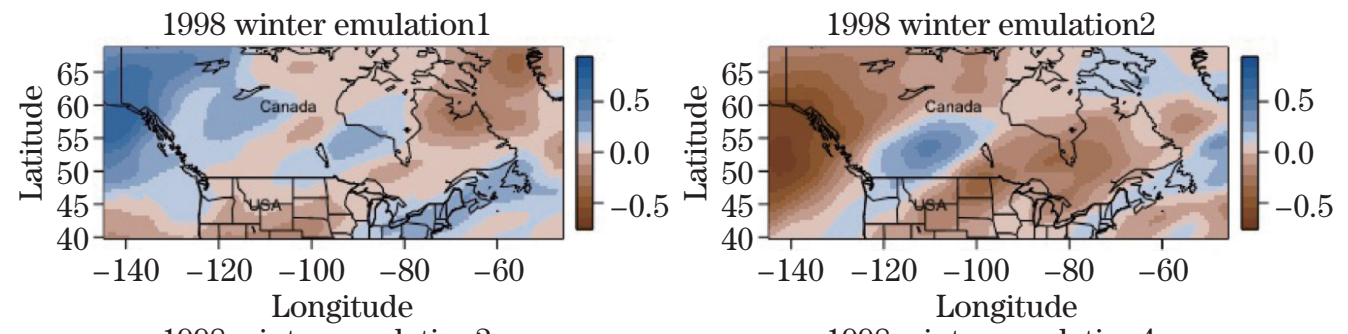

1998 winter emulation3
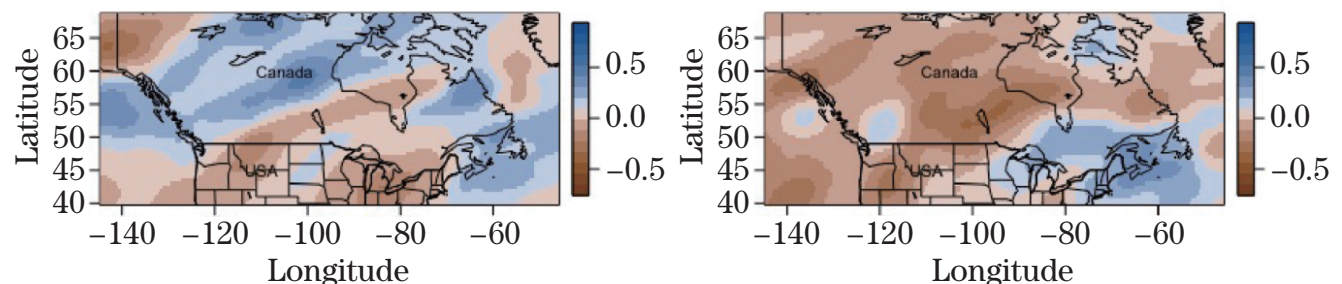

1998 winter emulation5
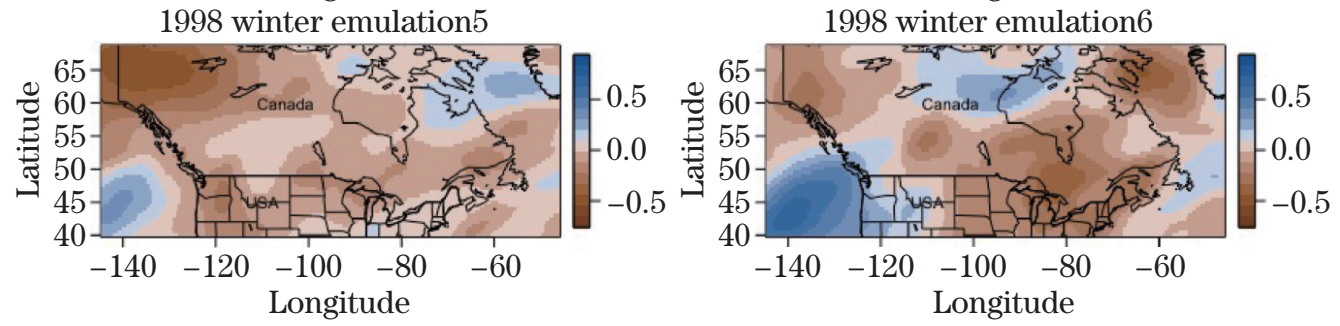

Figure 5. Six independent emulations of the residuals of winter precipitation rate in 1998 from our high-resolution simulation method.

shows the values of $D_{1 k}, k=1, \ldots, 4$, which describe the changes in the spatially varying parameters for each subregion in a given season. In terms of both spatial ranges, $\lambda_{1}$ and $\lambda_{2}, \hat{D}_{11}$ tends to be higher in the summers and $\hat{D}_{14}$ is higher in the winters.

We then implement the high-resolution simulation method to simulate the precipitation data at the RCM scale, with a total of 13,020 locations (longitude $\times$ latitude $=210 \times 62$ ) during summer and 11,484 locations (longitude $\times$ 


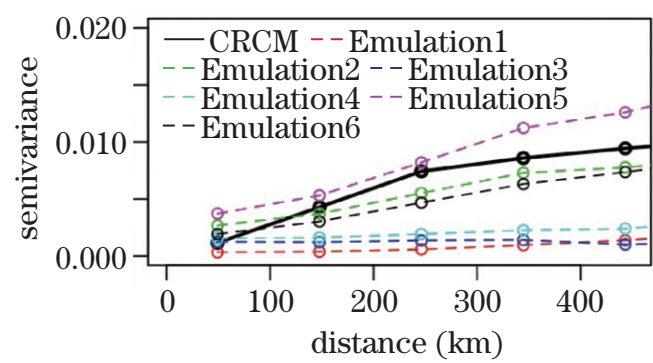

(a) 1998 winter local region1

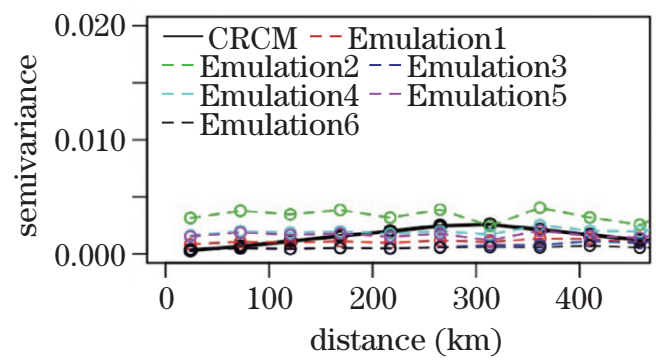

(c) 1998 winter local region3

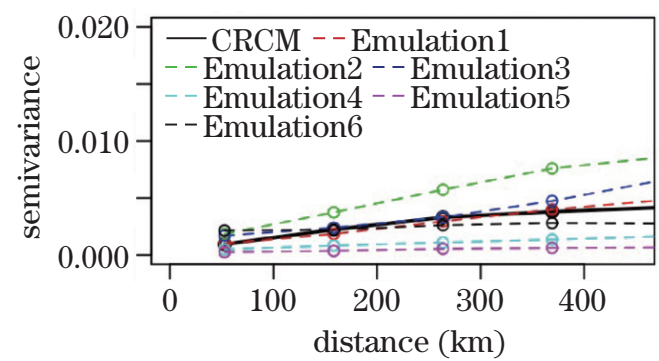

(b) 1998 winter local region2

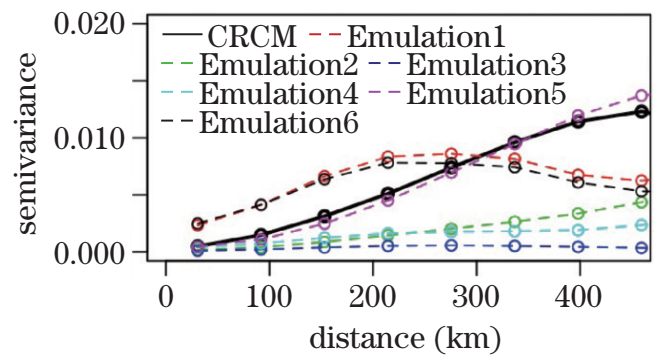

(d) 1998 winter local region4

Figure 6. Empirical variograms of four local regions of RCM runs and our emulations. The ranges of the four local regions are (a) from $\left(19.45^{\circ} \mathrm{N}, 130.00^{\circ} \mathrm{W}\right)$ to $\left(43.00^{\circ} \mathrm{N}\right.$, $\left.157.71^{\circ} \mathrm{W}\right)$, (b) from $\left(62.00^{\circ} \mathrm{N}, 130.00^{\circ} \mathrm{W}\right)$ to $\left(70.26^{\circ} \mathrm{N}, 157.71^{\circ} \mathrm{W}\right)$, (c) from $\left(19.45^{\circ} \mathrm{N}\right.$, $\left.35.72^{\circ} \mathrm{W}\right)$ to $\left(43.00^{\circ} \mathrm{N}, 53.00^{\circ} \mathrm{W}\right)$, and (d) from $\left(62.00^{\circ} \mathrm{N}, 53.00^{\circ} \mathrm{W}\right)$ to $\left(35.72^{\circ} \mathrm{W}\right.$, $\left.70.26^{\circ} \mathrm{N}\right)$.

latitude $=198 \times 58$ ) during winter. From west to east, we sequentially simulate a subset, including around $10 \%$ of the locations for each step, conditioning on around $20 \%$ of the simulated data in the previous step on the eastern boundary. Our six independent emulations for the residuals of the winter precipitation rate in 1998 are displayed in Figure 5.

Visually, from Figure 5, we can see that our emulations share common variance and spatial ranges with the RCM run for winter 1998. For instance, we see similar variabilities overall and clusters in the coastal areas along the Pacific Ocean and Greenland. Furthermore, we select four local regions in the corners and show the empirical variograms based on the RCM runs and our emulations in Figure 6. As shown, the variograms are significantly different in the local regions, which is evidence of spatial nonstationarity. In addition, the variograms of the RCM run can be viewed as a realization from our emulated ones. These results suggest that our model can provide similar statistical properties to the RCM, although the RCM run exhibits more local and regional features. 


\section{Discussion}

In this study, we have proposed a flexible method to model the spatially nonstationary covariance function, including the local stationary and weighted local stationary models as special cases. The proposed model allows more complex nonstationary features, with interpretable parameterization that characterizes the degree of nonstationarity. We have also developed an efficient estimation approach and validated its performance using simulation studies. Motivated by the computational issue of climate model downscaling, we have developed a fast high-resolution simulation method. Compared with RCM runs from traditional downscaling methods as the benchmark, our model captures similar spatial nonstationarity in a more efficient way.

In contrast to the full likelihood method, which estimates parameters directly, our methodology approximates global nonstationary covariance parameters by smoothing independently estimated local covariance parameters. In our work, the fundamental theory is the Taylor approximation. Similar to a nonparametric function estimation, we use a local polynomial representation for the spatially varying parameter. This is also different from the usual local polynomial estimator in a nonparametric regression model, where the approximation is of the mean function itself. After estimating all unknown coefficients, we are able to plot and visualize the estimated spatially varying parameter as a smooth function in space.

Our methodology can be also embedded into the Bayesian hierarchical framework, where the polynomial terms can be viewed as hyperparameters of the spatially varying parameters. However, these parameters, which are in the covariance function, are usually very sensitive to the priors, and sampling from a multilayer hierarchical model is computationally expensive.

In terms of high-resolution simulation, the method in Kleiber (2016) is more efficient, using the circulate embedding technique. For example, in Section 3.3 of Kleiber (2016), simulating a realization at a grid of $120 \times 98=11,760$ locations needs approximately 1 second, whereas around 15 minutes are required for our methods. However, this technique needs to estimate a spatial deformation function and then simulate data at regularly spaced locations. Therefore, it only works for regularly spaced spatial data, while ours is suitable for both regularly and irregularly spaced data.

For our future work, the nonstationarity index mentioned in Section 4.2 can be further extended to build a nonstationarity test. The null hypothesis is just 
whether the slope parameters are zeros. This can be achieved either using a likelihood ratio test based on the maximized likelihood ratio between WS0 and NS1, or using the Wald test based on the estimations of slope parameters. Future research is also needed to develop automatic and adaptive partitioning criteria. Although our models are less sensitive to region partitioning than the weighted local stationary model, using validation tools or the information from covariates (see Risser et al. (2016)) could further help optimize the region partition.

\section{Supplementary Materials}

Section S1 provides further details on the computations. Section S2 mentions more simulation studies. Section S3 includes additional figures. Section S4 gives the $\mathrm{R}$ source code for the simulation studies and applications. Section S5 provides the GCM and RCM data sources, including instructions on how to obtain related data.

\section{Acknowledgment}

This research was supported by funding from King Abdullah University of Science and Technology (KAUST). We would like to thank the editor, associate editor, and two anonymous reviewers for their valuable comments.

\section{References}

Anderes, E. B. and Stein, M. L. (2008). Estimating deformations of isotropic Gaussian random fields on the plane. The Annals of Statistics 36, 719-741.

Anderes, E. B. and Stein, M. L. (2011). Local likelihood estimation for nonstationary random fields. Journal of Multivariate Analysis 102, 506-520.

Banerjee, S., Gelfand, A. E., Finley, A. O. and Sang, H. (2008). Gaussian predictive process models for large spatial data sets. Journal of the Royal Statistical Society: Series B (Statistical Methodology) 70, 825-848.

Cressie, N. (2015). Statistics for Spatial Data. John Wiley \& Sons.

Das, S. and Nason, G. P. (2016). Measuring the degree of non-stationarity of a time series. Stat 5, 295-305.

Fouedjio, F., Desassis, N. and Rivoirard, J. (2016). A generalized convolution model and estimation for non-stationary random functions. Spatial Statistics 16, 35-52.

Fredericks, A. K. and Newman, K. B. (1998). A comparison of the sequential gaussian and markov-bayes simulation methods for small samples. Mathematical Geology 30, 1011-1032.

Gelfand, A. E. and Schliep, E. M. (2016). Spatial statistics and Gaussian processes: a beautiful marriage. Spatial Statistics 18, 86-104.

Gneiting, T., Ševčíková, H., Percival, D. B., Schlather, M. and Jiang, Y. (2006). Fast and 
exact simulation of large Gaussian lattice systems in $\mathbb{R}^{2}$ : exploring the limits. Journal of Computational and Graphical Statistics 15, 483-501.

Gotway, C. and Rutherford, B. (1994). Stochastic simultation for imaging spatial uncertainty: Comparison and evaluation of available algorithms. In: Geostatistical Simulations. Springer, pp. 1-21.

Gramacy, R. B. and Lee, H. K. H. (2008). Bayesian treed Gaussian process models with an application to computer modeling. Journal of the American Statistical Association 103, 1119-1130.

Higdon, D. (1998). A process-convolution approach to modelling temperatures in the North Atlantic Ocean. Environmental and Ecological Statistics 5, 173-190.

Jun, M. and Stein, M. L. (2008). Nonstationary covariance models for global data. The Annals of Applied Statistics 2, 1271-1289.

Kleiber, W. (2016). High resolution simulation of nonstationary Gaussian random fields. Computational Statistics \& Data Analysis 101, 277-288.

Königsberger, K. (2013). Analysis 2. Springer-Verlag.

Li, Y. and Zhu, Z. (2016). Modeling nonstationary covariance function with convolution on sphere. Computational Statistics \& Data Analysis 104, 233-246.

Lindgren, F., Rue, H. and Lindström, J. (2011). An explicit link between Gaussian fields and Gaussian Markov random fields: the stochastic partial differential equation approach. Journal of the Royal Statistical Society: Series B (Statistical Methodology) 73, 423-498.

Nychka, D., Bandyopadhyay, S., Hammerling, D., Lindgren, F. and Sain, S. (2015). A multiresolution Gaussian process model for the analysis of large spatial datasets. Journal of Computational and Graphical Statistics 24, 579-599.

Nychka, D., Wikle, C. and Royle, J. A. (2002). Multiresolution models for nonstationary spatial covariance functions. Statistical Modelling 2, 315-331.

Paciorek, C. J. and Schervish, M. J. (2004). Nonstationary covariance functions for Gaussian process regression. In: Advances in Neural Information Processing Systems. pp. 273-280.

Paciorek, C. J. and Schervish, M. J. (2006). Spatial modelling using a new class of nonstationary covariance functions. Environmetrics 17, 483-506.

Patterson, H. and Thompson, R. (1975). Maximum likelihood estimation of components of variance. In: Proceedings of the 8th International Biometric Conference. pp. 197-207.

Risser, M. D. (2016). Review: Nonstationary spatial modeling, with emphasis on process convolution and covariate-driven approaches. arXiv preprint arXiv:1610.0244\%.

Risser, M. D. and Calder, C. A. (2015a). Local likelihood estimation for covariance functions with spatially-varying parameters: the convoSPAT package for $\mathrm{R}$. arXiv preprint arXiv:150\%.08613.

Risser, M. D. and Calder, C. A. (2015b). Regression-based covariance functions for nonstationary spatial modeling. Environmetrics 26, 284-297.

Risser, M. D., Calder, C. A., Berrocal, V. J. and Berrett, C. (2016). Nonstationary spatial process modeling via treed covariate segmentation, with application to soil organic carbon stock assessment. arXiv preprint arXiv:1608.05655.

Sampson, P. D. and Guttorp, P. (1992). Nonparametric estimation of nonstationary spatial covariance structure. Journal of the American Statistical Association 87, 108-119.

Scinocca, J. F. and McFarlane, N. A. (2004). The variability of modeled tropical precipitation. Journal of the Atmospheric Sciences 61, 1993-2015. 
Shrestha, R. R., Schnorbus, M. A., Werner, A. T. and Zwiers, F. W. (2014). Evaluating hydroclimatic change signals from statistically and dynamically downscaled GCMs and hydrologic models. Journal of Hydrometeorology 15, 844-860.

Stein, M. L. (2005). Nonstationary spatial covariance functions. Unpublished report.

Sun, Y. and Genton, M. G. (2011). Functional boxplots. Journal of Computational and Graphical Statistics 20, 316-334.

Wilby, R. L. and Wigley, T. (1997). Downscaling general circulation model output: a review of methods and limitations. Progress in Physical Geography 21, 530-548.

Wood, A. T. and Chan, G. (1994). Simulation of stationary Gaussian processes in $[0,1]^{d}$. Journal of Computational and Graphical Statistics 3, 409-432.

King Abdullah University of Science and Technology, CEMSE Division, Thuwal 23955-6900, Saudi Arabia.

E-mail: yuxiao.li@kaust.edu.sa

King Abdullah University of Science and Technology, CEMSE Division, Thuwal 23955-6900, Saudi Arabia.

E-mail: ying.sun@kaust.edu.sa

(Received December 2017; accepted September 2018) 